\title{
对信访档案管理的几点思考
}

\author{
刘志和 \\ 吉林省洮南市信访局 \\ DOI:10.32629/ej.v1i4.72
}

[摘要] 信访档案不仅反映了信访工作的全貌,也呈现出了矛盾纠纷发生、发展以及平息的规律, 随着信访量的不断增加,把握 这些规律,加强信访档案管理,维护社会和谐就成为信访工作者面临的新课题。文章主要对信访档案管理工作提出了几点思考, 以供借鉴。

[关键词] 信访档案；管理工作；矛盾纠纷

信息时代的到来, 为信访工作也带来的一定变革, 要想 适应社会发展, 就需要对信访档案管理模式进行优化, 采用 先进的信息管理平台, 加强档案管理, 进而完善档案管理水 平, 有效解决群众中存在的问题, 为构建和谐社会而努力。

\section{1 信访档案及其特征}

信访档案同信访工作之间有着紧密联系, 信访档案是信 访活动的真实记录, 也是信访工作的重要依据。信访档案通 常被归类在民生档案中, 除了具有信息性、价值性、记录性 等基本特征外, 还具有一定的公正性, 可以说是人民矛盾解 决的关键见证。

信访档案是群众信访案件处理全过程的重要记录, 是在 开展信访工作中不可或缺的重要信息资源。一份较为完整的 信访档案中, 会详细的标注事件发生的时间、地点、关联人 物以及相关部门的信息等内容, 这为后续调查处理提供了基 础资料, 具有一定的准确性和参考价值。

\section{2 信访档案工作的重要性}

信访档案工作是信访工作中的关键内容, 具有一定的不 可替代性。通过信访档案工作的开展能够将固定时期内事业 单位, 或者组织的政策行为和结果真实准确的反映出来, 帮 助领导人员更加清晰的了解该区域发展的实际情况, 掌握信 访规律, 从而为现存问题制定合理的解决对策, 简化人民内 部矛盾, 促进区域内的协同发展。

信访档案工作会将各个时期内信访活动以及问题解决 情况进行详细记录, 且具有一定的真实性, 相关人员可以从 这些信访档案中借鉴和学习相应的经验, 从而为后续工作的 开展提供帮助, 增强决策等内容制定的合理性, 全面解决人 民存在的矛盾, 推动和谐社会的建设。

由于在信访档案中详细记录了信访者提供了数据、观点 以及组织调查结果, 通过对信访档案的查阅可以还原当时事 件真相, 并起到一定的借鉴作用, 从而为后期同类问题的处 理提供帮助。同时信访档案也为重信重访、越级信访等处理 提供了历史凭证。这对于一些尚未解决的信访事件来说有着 重要作用。只有具备完整齐备的信访档案, 才能将死档编程, 从根本上解决问题。

\section{3 信访档案管理的建议和对策}

信访档案作为处理人们来电、来访的重要历史记录, 是 信访工作开展中较为重要的资料和财富, 为了保证信访工作 的质量, 有必要加强对信访档案的管理, 充分发挥信访档案 作用, 实现我国建设目标。在信访档案管理中需要注意的重 点内容有:

3.1 确保信访档案收集的完整性

档案管理具有一定的复杂性和综合性, 其涉及的业务内 容较多, 所以在开展档案管理工作时, 要先对管理人员自身 的能力进行培训和考核, 丰富员工知识体系, 确保档案管理 工作内容的落实, 增大档案管理和利用率。另外, 在信访档案 管理中, 还要做好相应的收集工作, 这样才能增强档案的完 整性, 为后期工作处理提供充足的证据支持。传统的信访档 案管理大多都是在接到信访内容后, 责令相关部门开展案件 调查工作, 并将调查结果以书面形式传达给信访人, 在这一 过程中, 只有事件的接受和处理结果信息, 却没有案件处理 过程的信息, 这使得信访档案存在一定的不完整性, 为后期 利用带来的一定困难。为此, 要将基层信访案件处理过程中 所产生的相关资料进行及时收集和整理, 完善信访档案内 容。并在资料收集过程中, 注重资料的价值, 避免无用数据的 混入, 进而影像档案的有效性和真实性。

根据我国制定的相关要求, 完整的信访档案资料包括了 七方面内容:（1）上访信或者上访人基础信息登记表; (2)事 件初期处理部门的相关意见书; (3) 初期处理部门领导的复查 意见书; (4) 审核意见书; (5) 上访诉求问题的相关查办报告; (6) 在同上访人进行事件了解过程中的相关记录信息以及送达 回执; (7) 处理意见、复核意见、复核意见会议记录、纪要、 调查报告和相对应的国家政策及相关文件、佐证材料。(8) 在 案件交办过程中所形成的案件结案报告单。

\section{2 整理信访档案内容}

档案整理是档案管理工作中较为重要的一个环节, 具有 着承上启下的作用。在档案收集完成后, 要对收集到的相关 信息进行有效整理和规制, 并按照相关要求进行编号归档保 存。在档案整理过程中, 注重档案形成的规律, 保持文件资 料、结案报告、处理意见之间的历史联系。并从中选出有参 考价值的上访案件进行立卷归档, 以确保档案自身的历史和 
实用价值。另外, 整理和保存档案时, 应该尊重前人的劳动, 充分利用原有的整理基础, 注意分清哪些是合理的, 凡是合 理的都应继承下来, 这样有利于在以后工作中加以借鉴。

在整理信访档案的过程中, 不要随意打乱原有档案的顺 序, 对于一些已经立卷, 且理据清晰的信访档案应保持其原 有的整理体系。另外, 在立卷整理过程中, 需按照事件发生时 间、材料性质分开保管。一般情况下, 上级下发的材料文件 都是按照事件顺序立卷的, 其保质期相对较长。如果在档案 整理过程中, 发现其存在跨年情况, 需要按照案件终结时间 对其实施分类整理, 且按照长期和永久分开完成标号, 做到 及时归档立卷, 保证信访档案管理的规范化和制度化。

\section{3 确保信访档案的完整性和安全性}

信访档案管理工作的目的就是增大档案的利用率, 为解 决人民内部矛盾提供帮助。所以在信访档案管理过程中, 要 确保档案的完整性和安全性, 从而为档案的长远利用提供便 利。在档案管理工作中, 注重档案保护与利用之间的协调性, 不可为了实现其中一个目标而导致另一项出现问题, 进而影 响信访档案的有效性。为此, 在信访档案管理过程中, 需做到 防盗、防火、防水、防破坏、防尘、防霉变、防鼠、防虫、 防光这十项内容。

具体来说, 在信访档案管理过程中, 要设置专门的档案 保管设施, 并加大监管力度, 避免人为破坏的产生。且在保管 档案的区域内要做好防火、防水、防潮、防尘等的处理, 设 置合理的保护措施, 控制保管环境内的湿度, 确保档案材质 的干燥、整洁, 减少霉变、腐烂等问题的出现。此外, 还应该 在档案保管柜中放置樟脑球或者喷酒杀虫剂, 避免虫害对档 案的侵蚀。又由于纸质档案较多, 所以需避免阳光的直接照 射, 以免出现褪色、变黄发脆等问题, 影响信访档案的质量。

3. 4 明确信访档案的具体作用, 规范信访档案管理工作

第一, 信访档案具有凭证作用。信访档案具有一定的规 律和独特性, 其是当时事件发生的真实记录, 不存在编造现 象, 其具有一定的法律效益, 处理人员会根据记录的详细资 料来判定事件情况, 进而采取有针对性的处理措施。与此同 时信访档案也是初访演变的历史凭证, 是处理重访以及老客 户上访最为重要的依据。通过调阅信访档案, 能够帮助工作 人员了解事件处理情况, 并结合现有问题制定更为完善的解 决措施, 以缓解消极情绪的产生, 维护人们的资深利益, 进而
为和谐社会构建提供帮助。

第二, 信访档案具有参考作用。在案件处理过程中, 信访 档案具有真实性和可靠性特征。信访案件不都是实时的, 一 些案件因为涉及的因素较多, 再加上受到意识水平以及国情 等的限制, 使得案件解决起来需要较长时间, 甚至有可能会 涉及到近期政策的实施, 所以具有一定的延后性, 而信访档 案的存在则能够为案件处理提供充足的参考, 即使间隔时间 再长, 也能将当时事件发生原委呈现出来, 这样就为处理部 门工作的开展提供了帮助, 避免了随意性和失误问题的产 生。再者, 信访档案也为案件的进一步研究、工作总结和改 进提供了材料支持, 对于提高信访工作质量奠定了基础。

3.5 加强信息建设, 构建完善的信访档案大数据管理系统

信息时代影响下, 电子文件形式的信访档案逐渐替代了 原有大规模的纸质文件内容, 推动了档案管理逐渐向着数字 化、体系化的方向发展。而构建完善的信访档案数据库管理 系统, 就成为目前档案管理工作开展的首要任务。通过大数据 管理系统的构建, 一方面能够对用户需求进行准确定位。大数 据系统的构建可以改善数据信息获取效率, 并通过细致的分 析和挖掘, 找出用户的具体需求, 进而提供合理的服务内容, 提高信访工作质量。另一方面, 为用户提供更多优质的服务。 大数据系统的建立实现了非结构性数据的有效分析, 有效加 强了信访档案服务的个性化和精确化。此外, 受到大数据时代 的影响, 对于信访档案管理人员也有了严格的要求, 除了要招 募专业性较强的工作人员外, 还需对人员实行专业培训, 以完 善管理人员的服务水平。

\section{4 结束语}

综上, 信访档案是事业发展进程的历史记录, 对于总结 检验、筹划未来有着十分重要的作用, 只有加深对信访档案 管理的认识, 维护单位服务对象的利益, 才能为稳定社会做 贡献, 最终促进信访档案自身作用的全面发挥。

\section{[参考文献]}

[1]刘翩翩.信访工作中的档案管理创新思考[J].现代企 业文化,2016(21):114.

[2]李峰珍.浅谈对信访档案工作的思考[J]. 赤子(上中 旬),2014(09):196。

[3]李泽龙.浅谈如何加强信访档案管理 [J]. 民营科 技,2018(12):259. 